\title{
Einfluss verschiedener Unterlagen auf vegetative und generative Parameter bei der Apfelsorte 'Topaz' unter biologischen Anbaubedingungen in Ostösterreich
}

\author{
Andreas Spornberger ${ }^{1}$ Elisabeth Schüller ${ }^{1}$ Edina Vidéki ${ }^{2}$ György Végvári ${ }^{2}$
}

Eingegangen: 26. Januar 2017 / Angenommen: 2. November 2017 / Online publiziert: 29. November 2017

(c) Der/die Autor(en) 2017. Dieser Artikel ist eine Open-Access-Publikation.

Zusammenfassung Im Herbst 2008 wurde im Versuchszentrum der Universität für Bodenkultur in Wien die Apfelsorte 'Topaz' auf den Unterlagen M9 (Klon T337) mit und ohne Zwischenveredlung 'Rubinola', M26, M7 normal (auf $25 \mathrm{~cm}$ ) und hoch (auf $55 \mathrm{~cm}$ ) veredelt, MM111 und 'Bittenfelder Sämling' gepflanzt, als Spindel erzogen und acht Jahre unter biologischen Anbaubedingungen getestet. Die Veredlungen auf Sämling wuchsen am stärksten, gefolgt von MM 111 und M7 normal veredelt; im Vergleich schwächer wuchsen die auf M7 hoch und auf M26 veredelten Bäume, am schwächsten wuchsen die Bäume auf M9. Baumausfälle waren nur bei M9 ohne Zwischenveredlung zu beobachten. Die höchsten Erträge pro Hektar gab es bei M7 normal veredelt, gefolgt von M26, M7 hoch veredelt und M9 ohne Zwischenveredlung. Die auf M7 hoch veredelten Bäume wiesen das höchste mittlere Einzelfruchtgewicht auf, gefolgt von MM 111, am unteren Ende lagen M9 ohne und mit Zwischenveredlung und M26. Die Unterlage M7 mit Hochveredlung verbindet einen mittleren Wuchs mit hohem Ertrag und guter Fruchtqualität und kann daher für unseren trockenen Standort empfohlen werden. M9 brachte ohne Zwischenveredlung einen höheren spezifischen Ertrag und eine höhere Fruchtfleischfestigkeit nach der Lagerung im Vergleich zur Variante mit Zwischenveredlung. Inwieweit diese Vorteile bei 'Topaz' auf M9 mit einer Hochveredlung zur Regulierung von Phytophthora

Andreas Spornberger

andreas.spornberger@boku.ac.at

1 Department of Crop Sciences, University of Natural Resources and Life Sciences, Gregor Mendel Str. 33, 1180 Wien, Österreich

2 Faculty of Horticulture, Szent István University, Budapest, Ungarn cactorum kombiniert werden können, müsste in weiteren Forschungen untersucht werden.

Schlüsselwörter M9 · Zwischenveredlung · M26 · M7 · M111 · Sämling · 'Topaz' · Apfel

Influence of Rootstocks on Vegetative and Generative Characteristics of the Apple Cultivar 'Topaz' According to Organic Production Conditions in Eastern Austria

Abstract In autumn 2008, in the research orchard of the University of Natural Resources and Life Sciences in Vienna, the apple cultivar 'Topaz' was planted on the rootstocks M9 (clone T337) with and without 'Rubinola' as interstem, M26, M7 grafted at $25 \mathrm{~cm}$ and at $55 \mathrm{~cm}$ respectively, M111 and 'Bittenfelder seedling', trained as spindles and tested for eight years according to organic production rules. The trees on seeding grew very vigorously, followed by MM111 and M7 normally grafted; the M7 highly grafted trees showed less vigor, comparable with M26, while the trees on M9 with and without interstem grew very weakly. Tree losses were only observed on M9 without interstem. The highest yields per hectare were found on M7 normally grafted, followed by M26, M7 highly grafted and M9 without interstem. M7 highly grafted showed the highest single fruit weights, followed by MM111, at the other end were M9 with and without interstem, and M26. The rootstock M7 grafted at $55 \mathrm{~cm}$ combines a medium growth with high yield and good fruit quality and therefore can be recommended for dry areas like our site. M9 without interstem showed a higher specific yield and fruit firmness after storage compared to the variant with interstem. Whether these advantages for 'Topaz' on M9 can be combined with a higher 
grafting height as effective preventive method to control Phytophthora cactorum, has to be examined in further research.

Keywords M9 · Interstem · M26 · M7 · M111 - Seedling · 'Topaz' · Apple

\section{Problemstellung}

In den letzten Jahren ist der Einsatz stärker wüchsiger Apfel-Unterlagen, vor allem im extensiven und biologischen Anbau und für Nachbauflächen diskutiert worden (Ruess 2010; Hornig und Paul 2011). Diese verfügen über ein stärkeres Wurzelsystem als die Standardunterlage M9 und reagieren daher weniger sensibel auf Wühlmausfraß (Keppel et al. 1998). Außerdem sind sie besonders auf trockenen Standorten robuster gegenüber Trockenstress als die Standardunterlage M9 (Keppel et al. 1998). Im Zusammenhang mit dem Klimawandel könnten sie aufgrund ihres intensiveren Wurzelsystems in Zukunft vermehrt an Bedeutung gewinnen, da sie eine bessere Versorgung mit Wasser und Nährstoffen ermöglichen. Dies ist besonders für den biologischen Anbau wichtig, wo keine leicht löslichen Dünger zur Verfügung stehen.

In einem mehrjährigen Feldversuch wurde daher der Einfluss verschiedener mittel und stark wüchsiger Unterlagen auf vegetative und generative Parameter bei der in Österreich und Deutschland im biologischen Anbau weit verbreiteten Apfelsorte 'Topaz' im Vergleich zur Standardunterlage M9, auf einem trockenen Standort mit biologischer Bewirtschaftung untersucht.

\section{Material und Methoden}

Die Auspflanzung erfolgte im Versuchszentrum der Universität für Bodenkultur in Jedlersdorf, am nordöstlichen Stadtrand von Wien. Die Jahresdurchschnittstemperatur am Standort beträgt $10^{\circ} \mathrm{C}$, die durchschnittliche Niederschlagsmenge $550 \mathrm{~mm}$ im Jahr. Der Boden ist ein tiefgründiger, leichter Tschernosem auf Lösslehm mit hohem Kalkgehalt (ca. 15\%), die Bodenreaktion leicht alkalisch (pH 7,6). Im 2. Jahr und ab dem 4. Jahr erfolgte jährlich Ende März eine organische Düngung mit Biofert ( $30 \mathrm{~kg} \mathrm{~N} / \mathrm{ha}$ ).

Für den Versuch wurde die Sorte 'Topaz' von einer biologisch arbeitenden Baumschule aus der Steiermark (Deimel, Österreich) auf folgende Unterlagen veredelt: M9 (Klon T337) ohne und mit Zwischenveredlung (Sorte 'Rubinola' zwischen 20 und $65 \mathrm{~cm}$ ), M26, M7 (normal $=25 \mathrm{~cm}$ über dem Boden und hoch $=55 \mathrm{~cm}$ über dem Boden veredelt), MM111 und 'Bittenfelder Sämling'. Die einjährigen, vorwiegend unverzweigten Okulanten wurden im Herbst
2008 auf einer vorher ackerbaulich genutzten Fläche ausgepflanzt. Je Sorte und Unterlage standen 20 Bäume zur Verfügung, die in 5 Wiederholungsblöcken zu je 4 Bäumen randomisiert verteilt wurden. Jeder Wiederholungsblock wurde von beiden Seiten von einem Baum der Sorte 'Opal' auf M9 als Randbaum und Befruchter begrenzt. Erzogen wurde das im Anbau heute übliche Spindelsystem mit einem Reihenabstand von $4 \mathrm{~m}$; der Pflanzabstand in der Reihe variierte je nach Stärke der Unterlage zwischen 1,0 m bei M9 und M26, $1,5 \mathrm{~m}$ bei M7 und 1,8 $\mathrm{m}$ bei MM111 und Sämling.

Der Baumstreifen wurde mit einem Stockräumer mehrmals im Jahr mechanisch freigehalten, zwischen den Reihen wurde gemulcht. Der Pflanzenschutz erfolgte nach den Richtlinien des biologischen Anbaus, wobei im Frühjahr Neem und Quassia sowie nach der Blüte vor allem Netzschwefel und Granulosevirus eingesetzt wurden. Bewässert wurde in der Vegetationszeit nach Bedarf mit einer mobilen Überkronenberegnung. Sonstige Pflegemaßnahmen wie Baumerziehung, Schnitt und Mulchen der Fahrgasse wurden bei allen Unterlagenkombinationen auf ähnliche Weise durchgeführt. Die Ernte erfolgte per Hand in einem Durchgang, wobei Gewicht und Fruchtzahl baumweise erfasst wurden. Da der Baumabstand je nach Wuchsstärke der Unterlage verschieden war, wurde der Ertrag anschließend auf kg pro ha umgerechnet. Im Winter wurde der Stammumfang bei jedem Baum mit einem Maßband in $40 \mathrm{~cm}$ Höhe gemessen und das Vorhandensein von Wurzeltrieben bonitiert. Nach der Bonitur wurden die Wurzeltriebe entfernt. Außerdem wurden nach dem 3. Jahr im November 2011 bei jedem Baum Triebanzahl ( 1 = wenig, $9=$ viele $)$ und Triebansatzwinkel $(1=$ flach, $9=$ steil $)$ jeweils an der Mittelachse beurteilt. Der spezifische Ertrag wurde als kumulierter Hektarbaumertrag bezogen auf die errechnete Stammquerschnittsfläche im Herbst 2015 dargestellt. Die Befallsbonitur der Bäume mit der Mehligen Apfelblattlaus (Dysaphis plantaginea) erfolgte jährlich im Juni anhand einer Boniturskala von 0 (kein Befall) bis 5 (extrem starker Befall). In den Jahren 2013 bis 2015 wurde unmittelbar nach der Ernte eine Qualitätsbonitur aller geernteten Früchte durchgeführt. 2015 wurde außerdem je eine Kiste mit $20 \mathrm{~kg}$ pro Variante und Wiederholung für eine Farbbonitur der Früchte verwendet.

Von 2012 bis 2015 wurden jedes Jahr zur Ernte 25 Früchte pro Unterlagen-Variante und Wiederholung entnommen und bei $4{ }^{\circ} \mathrm{C}$ im Kühlraum gelagert. Für die Untersuchungen im Labor wurden 5 Früchte pro Wiederholung (insgesamt 25 pro Variante) entnommen und jeweils an zwei Terminen nach zwei (November) und fünf (Februar) Monaten untersucht. Gemessen wurden der Fruchtformindex (errechnet aus Fruchtlänge ${ }^{2} /$ Fruchtbreite * Fruchtdicke), Fruchtfleischfestigkeit (Penetrometer Kraftmessgerät AFG 500N, Mecmesin, Australien), lösliche Trockensubstanz mit einem digitalen Refraktometer (Atago, Japan) in ${ }^{\circ}$ Brix bei $20^{\circ} \mathrm{C}$ 
(Khazaei et al. 2008) und Vitamin C-Gehalt (ReflectoQuant RQflex, Merck, Deutschland). Die titrierbare Säure wurde mit dem Gerät TitroLinealpha plus (Schott, Deutschland) nach OECD (2005) ermittelt, wobei $5 \mathrm{ml}$ Probenvolumen bis $\mathrm{zu} \mathrm{pH} \mathrm{8,1} \mathrm{mit} \mathrm{0,1} \mathrm{mol/L} \mathrm{NaOH} \mathrm{titriert} \mathrm{wurden} \mathrm{(Thy-}$ bo et al. 2006). Daraus wurde der Säuregehalt berechnet: $[\mathrm{g} / \mathrm{L}]$ Äpfelsäure $=[\mathrm{ml}] \mathrm{NaOH} * 0,67 * 2$. Das Zucker-Säureverhältnis wurde mit folgender Formel errechnet: Brix * 10/[g/L] Äpfelsäure (OECD 2005).

Die statistische Auswertung der Daten erfolgte mit Hilfe des Statistikprogramms SPSS (Version 21, IBM, Österreich). Nach Prüfung der Daten auf Varianzhomogenität und Normalverteilung wurde eine Varianzanalyse in Verbindung mit einem F-Test durchgeführt, um die Mittelwerte anschließend mittels Grenzdifferenz nach S-N-K zu beurteilen, wobei generell mit dem Signifikanzniveau $p<0,05$ gearbeitet wurde.

\section{Ergebnisse und Diskussion}

\section{Baumausfälle}

Bei der Unterlage M9 Standard ohne Zwischenveredlung fielen im Beobachtungszeitraum insgesamt drei Bäume (15\%) aus, bei allen anderen Varianten gab es keine Ausfälle.

'Topaz' gilt als sehr anfällig für Kragenfäule (Phytophthora cactorum), denn die Sorte hat die Eigenschaft, Luftwurzeln am Stamm zu bilden, die leicht mit den Zoosporen von $P$. cactorum vom Boden aus infiziert werden können (Lindner 2009). Deshalb wird in der Praxis für diese Sorte in Kombination mit M9 entweder eine Hochveredlung (mindestens 40-70 cm oberhalb der Bodenlinie) oder die Verwendung einer widerstandsfähigen robusten Sorte (z. B. 'Summerred', 'Rubinola') als Zwischenveredlung empfohlen (Lindner 2009).

Die im Beobachtungszeitraum einzig bei der standardmäßig unten veredelten Unterlage M9 aufgetretenen Baumausfälle $(15 \%)$ belegen die Anfälligkeit der Sorte auf M9 und die Richtigkeit der Empfehlung, eine Zwischenveredlung zu verwenden.

\section{Wurzeltriebe}

Nach vier Jahren (2012) wiesen knapp $28 \%$ der Sämlinge und $15 \%$ der hoch veredelten M7-Bäume Wurzeltriebe auf, bei M9 und M7 normal veredelt war ein einzelner Baum betroffen, die anderen Varianten blieben frei. 2013 zeigten jeweils $10 \%$ der Bäume bei den Unterlagen Sämling und M7 hoch veredelt Wurzeltriebe. In den Jahren 2014 und 2015 gab es dann nur bei zwei bzw. drei der hoch veredelten Bäume (es waren immer dieselben) auf M7 Wurzeltrie-
Tab. 1 Ergebnisse der Bonituren auf Wurzeltriebe in den Jahren 2012-2015 (\% Bäume mit Wurzeltrieben)

\begin{tabular}{lllllllll}
\hline & 2012 & $*$ & 2013 & $*$ & 2014 & $*$ & 2015 & $*$ \\
\hline M9 & 5,9 & a & 0,0 & a & 0,0 & a & 0,0 & a \\
M9 mit Zwi- & 0,0 & a & 0,0 & a & 0,0 & a & 0,0 & a \\
schenveredlung & & & & & & & & \\
M26 & 0,0 & a & 0,0 & a & 0,0 & a & 0,0 & a \\
M7 normal & 5,3 & a & 0,0 & a & 0,0 & a & 0,0 & a \\
M7 hoch & 15,0 & ab & 10,0 & a & 10,0 & a & 15,0 & b \\
MM111 & 0,0 & a & 0,0 & a & 0,0 & a & 0,0 & a \\
Bittenfelder & 27,8 & $\mathrm{~b}$ & 11,1 & a & 0,0 & a & 0,0 & a \\
Sämling & & & & & & & & \\
\hline
\end{tabular}

*Verschiedene Buchstaben zeigen signifikante Unterschiede (ANOVA mit nachfolgendem SNK-test, $p<0,05$ )

be (Tab. 1). Eine geringere Ausbildung von Wurzeltrieben ist von Seiten des Anbauers her erwünscht, denn bei Infektion mit Feuerbrand (Erwinia amylovora) können sie zum Absterben des Baumes führen (Aldwinckle et al. 2004).

Aufgrund dieser Beobachtungen kann auf eine etwas geringere Affinität auf der Unterlage M7 bei Hochveredlung geschlossen werden im Vergleich zur Normalveredlung. Dennoch hatte dies keinerlei Auswirkungen auf Wuchs, Ertrag oder auf eine erhöhte Disposition zu Baumausfällen.

\section{Krankheiten und Schädlinge}

Trotz einer jährlich durchgeführten Behandlung im Rotknospenstadium mit Neem war zwischen 2013 bis 2016 jedes Jahr ein leichter Befall mit Mehliger Apfelblattlaus (Dysaphis plantaginea) festzustellen. Bei den durchgeführten Befallsbonituren konnten keine markanten und über mehrere Jahre wiederholbaren Unterschiede zwischen den Unterlagen festgestellt werden (Tab. 2). Ansonsten wurden keine Krankheiten und Schädlinge beobachtet.

Tab. 2 Ergebnisse der Bonituren auf Mehlige Apfelblattlaus (Dysaphis plantaginea) in den Jahre 2013-2016. (Boniturskala von $1=$ kein Befall bis $9=$ extrem starker Befall)

\begin{tabular}{lllllllll}
\hline & 2013 & $*$ & 2014 & $*$ & 2015 & $*$ & 2016 & $*$ \\
\hline M9 & 2,29 & ab & 1,59 & ab & 3,53 & a & 2,82 & a \\
M9 mit Zwi- & 2,30 & ab & 1,60 & ab & 3,20 & a & 3,45 & a \\
schenveredlung & & & & & & & & \\
M26 & 2,37 & ab & 1,63 & ab & 2,37 & a & 3,79 & a \\
M7 normal & 3,11 & b & 1,21 & a & 3,53 & a & 4,00 & a \\
M7 hoch & 2,10 & ab & 2,35 & b & 3,40 & a & 3,80 & a \\
MM111 & 2,74 & ab & 1,63 & ab & 3,32 & a & 3,58 & a \\
Bittenfelder & 1,56 & a & 1,33 & a & 2,44 & a & 3,22 & a \\
Sämling & & & & & & & & \\
\hline
\end{tabular}

*Verschiedene Buchstaben zeigen signifikante Unterschiede (ANOVA mit nachfolgendem SNK-test, $p<0,05$ ) 
Tab. 3 Ergebnisse der Bonitur auf Triebanzahl und -ansatzwinkel der Bäume von der Mittelachse aus im November 2011

Tab. 4 Wuchsstärke (mm Stammumfang in $40 \mathrm{~cm}$ Höhe) jeweils am Ende des Jahres gemessen (Ende 2009 bis 2015)

\begin{tabular}{lllll}
\hline & Triebanzahl & & Triebansatzwinkel & \\
& $1=$ wenig 9 = viel & $*$ & $1=$ flach 9= steil & $*$ \\
\hline M9 & 3,12 & ab & 3,24 & $\mathrm{a}$ \\
M9 mit Zwischenveredlung & 2,70 & $\mathrm{a}$ & 3,30 & $\mathrm{a}$ \\
M26 & 2,89 & $\mathrm{a}$ & 4,00 & $\mathrm{~b}$ \\
M7 normal & 3,84 & $\mathrm{c}$ & 3,79 & $\mathrm{ab}$ \\
M7 hoch & 3,55 & $\mathrm{bc}$ & 3,75 & $\mathrm{ab}$ \\
MM111 & 3,63 & $\mathrm{bc}$ & 4,16 & $\mathrm{~b}$ \\
Bittenfelder Sämling & 4,06 & $\mathrm{c}$ & 4,11 & $\mathrm{~b}$ \\
\hline
\end{tabular}

*Verschiedene Buchstaben zeigen signifikante Unterschiede (ANOVA mit nachfolgendem SNK-test, $p<$ $0,05)$

\begin{tabular}{lllllllllllllll}
\hline & 2009 & $*$ & 2010 & $*$ & 2011 & $*$ & 2012 & $*$ & 2013 & $*$ & 2014 & $*$ & 2015 & $*$ \\
\hline M9 & 55 & $\mathrm{a}$ & 63 & $\mathrm{a}$ & 75 & $\mathrm{a}$ & 93 & $\mathrm{a}$ & 102 & $\mathrm{a}$ & 111 & $\mathrm{a}$ & 132 & $\mathrm{a}$ \\
M9 mit Zwi- & 57 & $\mathrm{a}$ & 61 & $\mathrm{a}$ & 73 & $\mathrm{a}$ & 91 & $\mathrm{a}$ & 101 & $\mathrm{a}$ & 118 & $\mathrm{a}$ & 131 & $\mathrm{a}$ \\
schenveredlung & & & & & & & & & & & & & & \\
M26 & 53 & $\mathrm{a}$ & 66 & $\mathrm{a}$ & 89 & $\mathrm{~b}$ & 116 & $\mathrm{~b}$ & 134 & $\mathrm{~b}$ & 150 & $\mathrm{~b}$ & 168 & $\mathrm{~b}$ \\
M7 normal & 63 & $\mathrm{~b}$ & 78 & $\mathrm{~b}$ & 102 & $\mathrm{c}$ & 136 & $\mathrm{c}$ & 160 & $\mathrm{c}$ & 182 & $\mathrm{c}$ & 205 & $\mathrm{c}$ \\
M7 hoch & 69 & $\mathrm{c}$ & 82 & $\mathrm{~b}$ & 99 & $\mathrm{c}$ & 129 & $\mathrm{c}$ & 142 & $\mathrm{~b}$ & 159 & $\mathrm{~b}$ & 179 & $\mathrm{~b}$ \\
MM111 & 52 & $\mathrm{a}$ & 68 & $\mathrm{a}$ & 95 & $\mathrm{bc}$ & 136 & $\mathrm{c}$ & 167 & $\mathrm{c}$ & 199 & $\mathrm{~d}$ & 221 & $\mathrm{~d}$ \\
Bittenfelder Säm- & 65 & $\mathrm{~b}$ & 83 & $\mathrm{~b}$ & 112 & $\mathrm{~d}$ & 157 & $\mathrm{~d}$ & 195 & $\mathrm{~d}$ & 227 & $\mathrm{e}$ & 277 & $\mathrm{e}$ \\
ling & & & & & & & & & & & & & &
\end{tabular}

*Verschiedene Buchstaben zeigen signifikante Unterschiede (ANOVA mit nachfolgendem SNK-test, $p<$ $0,05)$

\section{Wuchs}

Bei den im Jahr 2011 durchgeführten Beobachtungen war bei den stärker wüchsigen Unterlagen Sämling, MM111 und M7 eine höhere Anzahl an Trieben an der Mittelachse zu finden als bei M26 und M9. Die Seitentriebe der auf M9 veredelten Bäume wiesen signifikant flachere Abgangswinkel auf als auf den Unterlagen M26, MM111 und Sämling; M7 lag dazwischen (Tab. 3).

Bei den Wuchsmessungen waren in den ersten beiden Jahren nur wenige Unterschiede zu beobachten. Nach Ende des dritten Jahres (2011) zeigten die Sämlinge dann bereits den signifikant kräftigsten Wuchs. Die Bäume auf MM111 waren am Anfang relativ schwach und zeigten erst nach fünf Jahren den zweitstärksten Wuchs. Nach 7 Jahren an dritter Stelle, und zwar schwächer als MM111 waren die normal veredelten Bäume auf M7. Die Bäume auf M7 waren am Anfang sehr stark wüchsig, vor allem die hoch veredelten wuchsen aber dann in den weiteren Jahren deutlich schwächer und lagen nach sieben Jahren auf gleichem Niveau wie M26, signifikant hinter den auf M7 normal veredelten. Abgesichert schwächer als alle anderen wuchsen beide M9-Varianten, wobei es keinen Unterschied machte, ob die Bäume mit oder ohne Zwischenveredlung gezogen wurden (Tab. 4).

\section{Ertrag}

In den Jahren 2010 und 2011 gab es erste, aber noch sehr geringe Erträge bei den schwach wüchsigen Unterlagen M9 und M26. Nennenswerte Erntemengen gab es dann im Jahr 2012 bei den Bäumen auf M9, M26 und beiden Varianten auf M7. Bei den stark wachsenden Unterlagen MM111 und Sämling konnten erst ab dem Jahr 2013 aussagekräftige Erträge festgestellt werden (Abb. 1).

In Summe der Jahre wiesen die Varianten M7 normal und M26 die höchsten Erträge pro Hektar auf, gefolgt von M7 hoch veredelt. M9 mit Zwischenveredlung hatte den

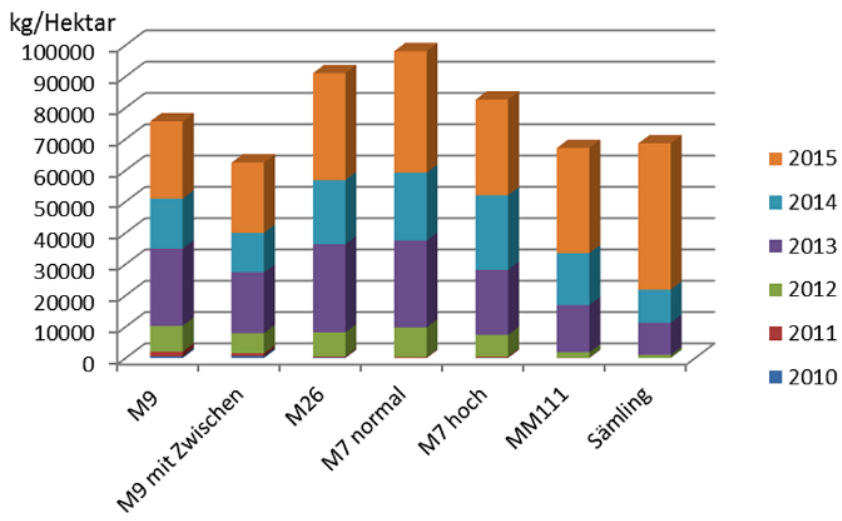

Abb. 1 Jährlicher Einzelbaumertrag (umgerechnet in $\mathrm{kg} / \mathrm{ha}$ ) von 2010 bis 2015 auf den verschiedenen Unterlagen-Varianten 
Tab. 5 Summe der Erträge in kg/ha von 2010-15, Stammquerschnitt nach 2015 und spezifischer Gesamtertrag von 'Topaz' auf den verschiedenen Unterlagen

\begin{tabular}{llllll}
\hline & $\begin{array}{l}\text { Summe } \\
2010-15\end{array}$ & $\begin{array}{l}\text { Stamm- } \\
\text { querschnitt } \\
\text { Ende 2015 }\end{array}$ & $\begin{array}{l}\text { Spezifischer } \\
\text { Gesamtertrag } \\
\text { pro Hektar } \\
\text { 2010-15 } \\
\mathrm{kg} / \mathrm{cm}^{2}\end{array}$ & $*$ \\
\hline M9 & kg/ha & $*$ & $\mathrm{~cm}^{2}$ & 5349 & $\mathrm{f}$ \\
M9 mit Zwischen- & 62439 & $\mathrm{a}$ & 13,57 & 4600 & $\mathrm{e}$ \\
veredlung & & & & & $\mathrm{d}$ \\
M26 & 90941 & $\mathrm{~cd}$ & 22,57 & 4029 & $\mathrm{~d}$ \\
M7 normal & 98032 & $\mathrm{~d}$ & 33,61 & 2917 & $\mathrm{c}$ \\
M7 hoch & 82554 & $\mathrm{bc}$ & 25,98 & 3178 & $\mathrm{c}$ \\
MM111 & 67103 & $\mathrm{a}$ & 39,86 & 1684 & $\mathrm{~b}$ \\
Bittenfelder Säm- & 68559 & $\mathrm{a}$ & 59,46 & 1153 & $\mathrm{a}$ \\
ling & & & & & \\
\hline
\end{tabular}

*Verschiedene Buchstaben zeigen signifikante Unterschiede (ANOVA mit nachfolgendem SNK-test, $p<0,05$ )

Tab. 6 Mittleres Stückgewicht in den Jahren 2013 bis 2015

\begin{tabular}{lllllll}
\hline & $\begin{array}{l}2013 \\
\mathrm{~kg}\end{array}$ & $*$ & $\begin{array}{l}2014 \\
\mathrm{~kg}\end{array}$ & $*$ & $\begin{array}{l}2015 \\
\mathrm{~kg}\end{array}$ & $*$ \\
\hline M9 & 0,135 & $\mathrm{a}$ & 0,176 & $\mathrm{a}$ & 0,123 & $\mathrm{a}$ \\
$\begin{array}{l}\text { M9 mit Zwi- } \\
\text { schenvered- }\end{array}$ & 0,143 & $\mathrm{abc}$ & 0,187 & $\mathrm{a}$ & 0,129 & $\mathrm{ab}$ \\
lung & & & & & & \\
M26 & 0,139 & $\mathrm{ab}$ & 0,175 & $\mathrm{a}$ & 0,129 & $\mathrm{ab}$ \\
M7 normal & 0,148 & $\mathrm{bc}$ & 0,188 & $\mathrm{a}$ & 0,140 & $\mathrm{bc}$ \\
M7 hoch & 0,152 & $\mathrm{c}$ & 0,182 & $\mathrm{a}$ & 0,150 & $\mathrm{c}$ \\
MM111 & 0,153 & $\mathrm{c}$ & 0,178 & $\mathrm{a}$ & 0,144 & $\mathrm{c}$ \\
Bittenfelder & 0,148 & $\mathrm{bc}$ & 0,184 & $\mathrm{a}$ & 0,151 & $\mathrm{c}$ \\
Sämling & & & & & & \\
\hline
\end{tabular}

*Verschiedene Buchstaben zeigen signifikante Unterschiede (ANOVA mit nachfolgendem SNK-test, $p<0,05$ )
Tab. 8 Ergebnisse der Bonitur der Fruchtausfärbung im Jahr 2015 (Prozentualer Anteil der Früchte in den Klassen)

\begin{tabular}{|c|c|c|c|c|c|}
\hline & \multicolumn{5}{|c|}{$\%$ Deckfarbe } \\
\hline & $<25$ & 25 bis 50 & 50 bis 75 & $>75$ & $*$ \\
\hline M9 & 1,8 & 10,9 & 22,1 & 65,2 & $\mathrm{~b}$ \\
\hline $\begin{array}{l}\text { M9 Zwischenver- } \\
\text { edlung }\end{array}$ & 3,8 & 9,0 & 22,4 & 64,8 & $\mathrm{~b}$ \\
\hline M 26 & 1,3 & 17,1 & 35,1 & 46,4 & $a b$ \\
\hline M7 hoch & 8,1 & 13,3 & 21,5 & 57,1 & $a b$ \\
\hline M7 normal & 15,3 & 21,3 & 24,9 & 38,5 & $a b$ \\
\hline MM 111 & 5,4 & 13,9 & 26,8 & 53,9 & $a b$ \\
\hline $\begin{array}{l}\text { Bittenfelder } \\
\text { Sämling }\end{array}$ & 16,6 & 24,9 & 31,4 & 27,1 & $\mathrm{a}$ \\
\hline
\end{tabular}

niedrigsten Gesamtertrag und war in Summe der 6 Jahre statistisch nicht von Sämling, MM111 und M9 Standard zu unterscheiden (Tab. 5).

Unter Berücksichtigung der Wuchsstärke wies die schwach wüchsige Unterlage M9 den höchsten spezifischen Ertrag auf, wobei die einmal veredelten Bäume (M9 Standard) sich signifikant von der Variante mit Zwischenveredlung absetzen konnten. Dahinter reihten sich M26, gefolgt von den beiden M7-Varianten. Deutlich darunter lagen MM111 und Sämling (Tab. 5).

Im Jahr 2013 wiesen MM111 (152 g) und M7 hoch veredelt $(153 \mathrm{~g})$ ein signifikant höheres Fruchtgewicht auf als M9 Standard (135 g) und M26 (139g); auf M7 normal veredelt (148 g) und Sämling (148 g) waren die Früchte auch noch größer als auf M9 Standard. M9 mit Zwischenveredlung (143g) war dagegen nicht unterscheidbar von den anderen. 2014 waren die Früchte insgesamt größer, und es gab keine Unterschiede zwischen den Varianten. 2015 zeigten Sämling (151 g), M7 hoch veredelt (150 g) und MM111 (144 g) höhere Werte im Vergleich zu M9 (123 g), M9 mit Zwischenveredlung und M26 (je 129g). Bei M7 normal veredelt $(140 \mathrm{~g})$ lag das mittlere Fruchtgewicht in diesem
Tab. 7 Ergebnisse der Qualitätsbonitur bei der Ernte 2015

\begin{tabular}{|c|c|c|c|c|c|c|c|c|c|c|}
\hline & $\begin{array}{l}\% \text { ver- } \\
\text { marktbar }\end{array}$ & $*$ & $\begin{array}{l}\text { \% Apfel- } \\
\text { wickler }\end{array}$ & $*$ & $\begin{array}{l}\% \\
\text { Laus }\end{array}$ & $*$ & $\begin{array}{l}\% \\
\text { Stippe }\end{array}$ & $*$ & $\begin{array}{l}\% \mathrm{zu} \\
\text { klein }\end{array}$ & $*$ \\
\hline$\overline{\mathrm{M} 9}$ & 88,0 & $\bar{a}$ & 0,3 & $\mathrm{a}$ & 3,5 & $\bar{a}$ & 0,1 & $\bar{a}$ & 7,9 & $\mathrm{c}$ \\
\hline $\begin{array}{l}\text { M9 mit Zwischen- } \\
\text { veredlung }\end{array}$ & 87,4 & $\mathrm{a}$ & 0,9 & $\mathrm{a}$ & 1,9 & $\mathrm{a}$ & 0,1 & $\mathrm{a}$ & 8,8 & $\mathrm{c}$ \\
\hline M26 & 92,6 & $\mathrm{a}$ & 0,6 & $\mathrm{a}$ & 2,0 & $\mathrm{a}$ & 0,2 & $\mathrm{a}$ & 4,0 & $\mathrm{~b}$ \\
\hline M7 normal & 88,0 & $\mathrm{a}$ & 1,0 & $\mathrm{a}$ & 9,4 & $\mathrm{c}$ & 1,1 & $a b$ & 0,2 & $\mathrm{a}$ \\
\hline M7 hoch & 88,8 & $\mathrm{a}$ & 0,8 & $\mathrm{a}$ & 7,8 & $\mathrm{bc}$ & 1,4 & $a b$ & 0,4 & $\mathrm{a}$ \\
\hline MM111 & 93,4 & $\mathrm{a}$ & 0,4 & $\mathrm{a}$ & 5,2 & $a b$ & 0,7 & $\mathrm{a}$ & 0,0 & $\mathrm{a}$ \\
\hline $\begin{array}{l}\text { Bittenfelder } \\
\text { Sämling }\end{array}$ & 88,2 & $\mathrm{a}$ & 0,6 & $\mathrm{a}$ & 7,4 & $\mathrm{bc}$ & 2,2 & $\mathrm{~b}$ & 0,7 & $\mathrm{a}$ \\
\hline
\end{tabular}

*Verschiedene Buchstaben zeigen signifikante Unterschiede (ANOVA mit nachfolgendem SNK-test, $p<$ $0,05)$ 
Tab. 9 Ergebnisse der von 2012 bis 2015 (Mittelwerte aus allen vier Jahren) durchgeführten Fruchtqualitätsmessungen im Labor jeweils nach zwei (November) bzw. fünf (Februar) Monaten Kühllagerung

\begin{tabular}{|c|c|c|c|c|c|c|c|c|c|c|c|c|}
\hline & $\begin{array}{l}\text { Frucht- } \\
\text { formindex }\end{array}$ & $*$ & $\begin{array}{l}\text { Fruchtfleisch- } \\
\text { festigkeit } \\
{\left[\mathrm{kg} / \mathrm{cm}^{2}\right]}\end{array}$ & $*$ & $\begin{array}{l}\text { Lösliche } \\
\text { TS } \\
{\left[{ }^{\circ} \text { Brix] }\right.}\end{array}$ & $*$ & $\begin{array}{l}\text { Äpfel- } \\
\text { säure } \\
{[\mathrm{g} / \mathrm{L}]}\end{array}$ & $*$ & $\begin{array}{l}\text { Zucker- } \\
\text { Säure- } \\
\text { Verhältnis }\end{array}$ & $*$ & $\begin{array}{l}\text { Vitamin C } \\
{[\mathrm{mg} / \mathrm{L}]}\end{array}$ & $*$ \\
\hline \multicolumn{13}{|l|}{ November } \\
\hline M9 & 0,57 & $\mathrm{a}$ & 5,89 & $\mathrm{a}$ & 14,96 & $\mathrm{a}$ & 9,10 & $\mathrm{a}$ & 17,01 & $\mathrm{a}$ & 140,07 & $\mathrm{a}$ \\
\hline $\begin{array}{l}\text { M9 mit Zwischenver- } \\
\text { edlung }\end{array}$ & 0,58 & $\mathrm{a}$ & 5,86 & $\mathrm{a}$ & 14,76 & $\mathrm{a}$ & 8,35 & $\mathrm{a}$ & 18,09 & $\mathrm{a}$ & 133,67 & $\mathrm{a}$ \\
\hline M26 & 0,58 & $\mathrm{a}$ & 5,89 & $\mathrm{a}$ & 15,14 & $\mathrm{a}$ & 8,51 & $\mathrm{a}$ & 18,38 & $\mathrm{a}$ & 136,07 & $\mathrm{a}$ \\
\hline M7 normal & 0,58 & $\mathrm{a}$ & 5,94 & $\mathrm{a}$ & 14,80 & $\mathrm{a}$ & 8,81 & $\mathrm{a}$ & 17,28 & $\mathrm{a}$ & 137,60 & $\mathrm{a}$ \\
\hline M7 hoch & 0,59 & $\mathrm{a}$ & 5,91 & $\mathrm{a}$ & 14,48 & $\mathrm{a}$ & 8,54 & $\mathrm{a}$ & 17,43 & $\mathrm{a}$ & 129,13 & $\mathrm{a}$ \\
\hline M111 & 0,61 & $\mathrm{a}$ & 6,02 & $\mathrm{a}$ & 14,84 & $\mathrm{a}$ & 9,15 & $\mathrm{a}$ & 16,88 & $\mathrm{a}$ & 151,60 & $\mathrm{a}$ \\
\hline Bittenfelder Sämling & 0,60 & $\mathrm{a}$ & 5,97 & $\mathrm{a}$ & 14,43 & $\mathrm{a}$ & 9,44 & $\mathrm{a}$ & 15,92 & $\mathrm{a}$ & 124,50 & $\mathrm{a}$ \\
\hline \multicolumn{13}{|l|}{ Februar } \\
\hline M9 & 0,58 & $\mathrm{a}$ & 5,10 & $\mathrm{c}$ & 15,43 & $\mathrm{a}$ & 7,01 & a & 22,60 & a & 148,53 & $\mathrm{a}$ \\
\hline $\begin{array}{l}\text { M9 mit Zwischenver- } \\
\text { edlung }\end{array}$ & 0,57 & $\mathrm{a}$ & 4,84 & $\mathrm{~b}$ & 15,22 & $\mathrm{a}$ & 6,95 & $\mathrm{a}$ & 22,36 & $\mathrm{a}$ & 150,33 & $\mathrm{a}$ \\
\hline M26 & 0,59 & $\mathrm{a}$ & 4,96 & $\mathrm{bc}$ & 15,50 & $\mathrm{a}$ & 7,00 & a & 22,52 & a & 148,93 & a \\
\hline M7 normal & 0,57 & $\mathrm{a}$ & 4,90 & $\mathrm{bc}$ & 15,31 & $\mathrm{a}$ & 7,07 & $\mathrm{a}$ & 22,41 & $\mathrm{a}$ & 133,27 & $\mathrm{a}$ \\
\hline M7 hoch & 0,57 & $\mathrm{a}$ & 4,87 & $\mathrm{~b}$ & 15,09 & $\mathrm{a}$ & 6,63 & $\mathrm{a}$ & 23,08 & a & 147,80 & $\mathrm{a}$ \\
\hline M111 & 0,59 & $\mathrm{a}$ & 4,91 & $\mathrm{bc}$ & 15,41 & $\mathrm{a}$ & 6,95 & a & 22,75 & a & 149,87 & $\mathrm{a}$ \\
\hline Bittenfelder Sämling & 0,59 & $\mathrm{a}$ & 4,65 & $\mathrm{a}$ & 14,79 & $\mathrm{a}$ & 6,77 & $\mathrm{a}$ & 22,36 & $\mathrm{a}$ & 130,60 & $\mathrm{a}$ \\
\hline
\end{tabular}

*Verschiedene Buchstaben zeigen signifikante Unterschiede (ANOVA mit nachfolgendem SNK-test, $p<0,05$ )

Jahr höher als M9 Standard, war aber nicht unterscheidbar von den anderen Varianten (Tab. 6).

Die Qualitätsbonitur der geernteten Früchte ergab in den Jahren 2013 und 2014 keine Unterschiede zwischen den Varianten (Ergebnisse nicht dargestellt). Im Jahr 2015 wurde ein höherer Anteil (je ca. $8 \%$ ) an zu kleinen Früchten in den M9-Varianten festgestellt, bei M26 waren es vergleichsweise weniger ( $4 \%$ ), bei den stärker wüchsigen Unterlagen dagegen gab es fast keine zu kleinen Früchte. Der Anteil an verlausten Äpfeln war bei den Unterlagen M7 (9,4 und $7,8 \%)$ und Sämling $(7,4 \%)$ deutlich höher im Vergleich zu M26 (2,0\%) und M9 (1,9 und 3,5\%). Beim Sämling $(2,2 \%)$ wurden auch mehr Früchte mit Stippe im Vergleich zu M9 (0,1\%), M26 (0,2\%) und MM111 (0,7\%) festgestellt.

Während bei der weiter oben dargestellten Bonitur der Mehligen Apfelblattlaus (Tab. 2) an den Bäumen im Sommer 2015 keine bedeutenden Unterschiede zwischen den Unterlagen festgestellt worden waren, war zur Ernte ein höherer Anteil an Lausäpfeln bei M7 und Sämling im Vergleich zu den schwächeren Unterlagen M26 und M9 festzustellen (Tab. 7). Vermutlich waren die Lausfrüchte bei den stärker wachsenden und dichteren Bäumen weniger gut sichtbar und daher bei der im Sommer durchgeführten Qualitätsausdünnung nicht exakt entfernt worden. Der stärkere Wuchs ist auch eine Erklärung für den höheren Anteil an Stippefrüchten beim Sämling.

\section{Fruchtqualität}

Die Bonitur der Ausfärbung im Jahr 2015 ergab einen signifikant höheren Anteil an gut ausgefärbten Früchten bei beiden M9-Varianten im Vergleich zum Sämling. Alle anderen Unterlagen lagen dazwischen und unterschieden sich nicht signifikant voneinander. Der starke und dichte Wuchs der Sämlinge wirkte sich somit auch nachteilig auf die Ausfärbung aus (Tab. 8).

Bei den Laboruntersuchungen nach zwei Monaten Lagerung im November wurden keine signifikanten Unterschiede festgestellt (Tab. 9 oben).

Bei den Untersuchungen im Februar nach fünf Monaten Lagerung fanden wir signifikante Unterschiede bei der Fruchtfleischfestigkeit. M9 Standard zeigte signifikant höhere Werte $\left(5,10 \mathrm{~kg} / \mathrm{cm}^{2}\right)$ als $\mathrm{M} 7$ hoch $\left(4,87 \mathrm{~kg} / \mathrm{cm}^{2}\right)$ und M9 mit Zwischenveredlung $\left(4,84 \mathrm{~kg} / \mathrm{cm}^{2}\right)$. Am signifikant niedrigsten lagen die Werte beim Sämling $\left(4,65 \mathrm{~kg} / \mathrm{cm}^{2}\right)$. Die übrigen Varianten lagen in der Fruchtfleischfestigkeit zwischen M9 Standard und M9 mit Zwischenveredlung, und waren von diesen nicht zu unterscheiden.

Keine signifikanten Unterschiede konnten bei Fruchtformindex, löslicher Trockensubstanz, Vitamin C- und Äpfelsäure-Gehalt und beim Zucker-Säure-Verhältnis gefunden werden (Tab. 9 unten). 


\section{Schlussfolgerungen}

Die beiden Varianten von M9 (mit und ohne Zwischenveredlung) zeigten in vielen Parametern (Wuchs, Schädlinge, Fruchtqualität, Deckfarbe) vergleichbare Ergebnisse. Unterschiede gab es aber z. B. bei den Baumausfällen. Ohne Zwischenveredlung fielen bei der Unterlage M9 in sieben Jahren $15 \%$ der Bäume aus, bei den Bäumen mit Zwischenveredlung gab es dagegen keine Ausfälle. Auf der anderen Seite hatten die Bäume mit Zwischenveredlung verglichen mit den Standard-Bäumen einen geringeren spezifischen Ertrag und nach 5-monatiger Lagerung eine niedrigere Fruchtfleischfestigkeit. Dass sich eine Zwischenveredlung beim Apfel nachteilig auf die Ertragsbildung auswirken kann, hatte schon Köksal (1973) festgestellt. Da 'Topaz' auf M9 ohne Zwischenveredlung veredelt zwar sehr anfällig für Kragenfäule ist, aber zugleich einen höheren spezifischen Ertrag und nach der Lagerung höhere Fruchtfestigkeit bringt, ist zu klären, ob eine Hochveredlung, wie sie Lindner (2009) neben der Zwischenveredlung als weitere Möglichkeit der Vorbeugung gegen Kragenfäule empfiehlt, nicht eine günstigere Alternative zur Zwischenveredlung darstellt.

Die Bäume auf M26 wiesen einen stärkeren Wuchs und damit verbunden höheren Einzelbaumertrag verglichen mit M9 auf. In vielen anderen Parametern, so auch im mittleren Fruchtgewicht, gab es dagegen keine Unterschiede zu M9. Da im Gegensatz zu M7 trotz stärkeren Wuchses keine Verbesserung der Fruchtgröße erzielt werden konnte, ist diese Unterlage keine überlegenswerte Alternative für M9.

Die Veredlungen auf M7 wuchsen vor allem in den ersten zwei Jahren sehr stark. Mit einsetzendem Ertrag wurde der Wuchs ab dem dritten Jahr schwächer. Die normal veredelten Bäume lagen nach sieben Jahren im Wuchs zwischen MM111 und M26, die hoch veredelten waren dagegen deutlich schwächer und mit M26 vergleichbar. Trotz eines im Vergleich zu M9 späteren Ertragsbeginns war der Einzelbaumertrag in Summe der sieben Jahre bei den normal veredelten M7-Bäumen am höchsten von allen Varianten. Die hoch veredelten wiesen aufgrund des schwächeren Wuchses etwas niedrigere Werte auf, waren aber im spezifischen Ertrag nicht von den normal veredelten zu unterscheiden. Zugleich waren die mittleren Fruchtgewichte bei dieser Unterlage in zwei Jahren deutlich höher als bei M9, vor allem bei den hoch veredelten. Letztere zeigten auch gute Werte hinsichtlich Ausfärbung und Fruchtqualität, lediglich die Fruchtfleischfestigkeit nach fünf Monaten Lagerung war geringer als bei M9 ohne Zwischenveredlung. Sotiropoulos (2006) hat ebenfalls die positiven Ertragseigenschaften von M7 (neben MM 106) bei einem Unterlagenversuch in Nordgriechenland hervorgehoben. Daher kann M7 bei Hochveredlung für 'Topaz' aufgrund ihrer Wuchsreduktion im Vergleich zur Normalveredlung verbunden mit hohem
Ertrag und Fruchtgewicht für diesen eher trockenen Standort empfohlen werden.

Bei der Unterlage MM111 wurde ähnlich wie beim Sämling ein später Ertragseintritt beobachtet. Insgesamt blieb diese Variante im summierten Einzelbaumertrag deutlich hinter den Unterlagen M26 und M7 zurück. Der spezifische Ertrag war zwar höher im Vergleich zum Sämling, aber auch deutlich geringer verglichen mit den anderen getesteten Typenunterlagen. Obwohl Fruchtausfärbung und Fruchtfleischfestigkeit verglichen mit dem Sämling besser waren, ist diese Unterlage aufgrund unserer Ergebnisse für einen Anbau in dieser Form nicht zu empfehlen.

Der Sämling war aus vergleichstechnischen Gründen in dem Versuch mitgelaufen, für die Praxis im Intensivanbau kommt die Unterlage aber nicht in Frage. Die bekannten Nachteile, wie der sehr starke Wuchs und damit verbunden spätere Ertragseintritt, sowie ein geringer spezifischer Ertrag wurden auch in diesem Versuch festgestellt. Außerdem gab es bei dieser Unterlage vergleichsweise viele Früchte mit Stippe und Schäden von der Mehligen Blattlaus, die Früchte waren weniger ausgefärbt und wiesen nach $5 \mathrm{Mo}-$ naten Lagerung eine signifikant niedrigere Fruchtfleischfestigkeit verglichen mit allen anderen Varianten auf.

Danksagung Dank gebührt der Baumschule Deimel in Ottendorf an der Rittschein für die kostenlose Zurverfügungstellung der Versuchsbäume

Funding Open access funding provided by University of Natural Resources and Life Sciences Vienna (BOKU).

Interessenkonflikt A. Spornberger, E. Schüller, E. Vidéki und G. Végvári geben an, dass kein Interessenkonflikt besteht.

Open Access Dieser Artikel wird unter der Creative Commons Namensnennung 4.0 International Lizenz (http://creativecommons.org/ licenses/by/4.0/deed.de) veröffentlicht, welche die Nutzung, Vervielfältigung, Bearbeitung, Verbreitung und Wiedergabe in jeglichem Medium und Format erlaubt, sofern Sie den/die ursprünglichen Autor(en) und die Quelle ordnungsgemäß nennen, einen Link zur Creative Commons Lizenz beifügen und angeben, ob Änderungen vorgenommen wurden.

\section{Literatur}

Aldwinckle H, LoGiudice N, Robinson TL, Holleran HT, Fazio G, Johnson WC, Norelli JL (2004) Resistance of apple rootstocks to fire blight infection caused by internal movement of Erwinia amylovora from scion infections. Acta Hortic 663:229-234

Hornig R, Paul M (2011) Rootstock-variety-combinations for juice apple production. Erwerbs-Obstbau 53:59-67

Keppel H, Pieber K, Weiss J (1998) Obstbau, Anbau und Verarbeitung, 2. Aufl. Leopold Stocker Verlag Graz, Stuttgart

Khazaei J, Chegini G, Bakhshiani M (2008) A novel alternative method for modelling the effect of air dry temperature and slice thickness on quality and drying kinetics of tomato slices: Superposition technique. Dry Technol 26:759

Köksal AI (1973) Wechselwirkungen zwischen Sorten, Unterlagen und Zwischenveredlungen beim Apfel: I. Vegetative und generative 
Leistungen der Kombinationen. Die. Gartenbauwissenschaft, Bd. 38., S 221-241

Lindner L (2009) Phytophthora cactorum als Verursacher von Rindenund Fruchtschäden am Apfel. Obstbau-Weinbau 9:312-315

OECD (2005) Internationale Normung von Obst und Gemüse, Leitfaden zu objektiven Testmethoden zur Bestimmung der Qualität von Obst und Gemüse sowie Trocken- und getrockneten Erzeugnissen. http://www.ble.de/SharedDocs/Downloads/02_Kontrolle/ 01_Qualitaetskontrolle/02_VermarktungsnormenObstGemuese/ LeitfadenQBestObstGemuese.pdf?_blob=publicationFile
Ruess F (2010) Intensiver Mostobstanbau. Obst und Garten 10:168-171 Sotiropoulos TE (2006) Delicious' grafted on five rootstocks in Northern Greece, Archives of Agronomy and Soil. of the apple cultivar ', Bd. 52. Performance, Golden, S 347-352

Thybo K, Edelenbos M, Christensen L, Sørensen J, Thorup-Kristensen K (2006) Effect of organic growing systems on sensory quality and chemical composition of tomatoes. Lwt Food Sci Technol 39:835 